\title{
Immediate recovery of the left atrial and left ventricular diastolic function after transcatheter aortic valve implantation: A transesophageal echocardiography study
}

\author{
Cenk Sarı ${ }^{1}$, Abdullah Nabi Aslan ${ }^{1}$, Serdal Baştuğ ${ }^{1}$, Murat Akçay², \\ Nihal Akar Bayram², Emine Bilen, Hüseyin Ayhan², Hacı Ahmet Kasapkara², \\ Tahir Durmaz ${ }^{2}$, Telat Keleş², Engin Bozkurt ${ }^{2}$ \\ ${ }^{1}$ Department of Cardiology, Ankara Ataturk Education and Research Hospital, Ankara, Turkey \\ ${ }^{2}$ Department of Cardiology, Yıldırım Beyazıt University, Faculty of Medicine, Ankara, Turkey
}

\begin{abstract}
Background: Chronic increased afterload due to severe aortic stenosis (AS) results in compensatory concentric left ventricular (LV) hypertrophy and LV dysfunction. These in turn cause remodeling of the left heart. The aim of this study was to investigate the acute effect of transcatheter aortic valve implantation (TAVI) on left atrial (LA) mechanics and LV diastolic function.
\end{abstract}

Methods: The study consisted of a total of 35 consecutive patients (mean age was $77.7 \pm$ \pm 5.0 years, 25 female) undergoing TAVI. All TAVI procedures have been performed under the transesophageal echocardiography (TEE) guidance. Before and 24 h after TAVI, all patients underwent transthoracic echocardiography (TTE) and mitral inflow velocities with pulsedwave $(P W)$ Doppler including early filling wave $(E)$, late diastolic filling wave $(A)$, and $E / A$ ratio were obtained. $L V$ diastolic function was also explored by pulsed tissue Doppler imaging (TDI). Early (E') and late (A') diastolic annular velocities, E'/A' ratio and E/E' ratio were obtained. In addition, during the procedure before and minutes after the valve implantation, the left atrial appendage-peak antegrade flow velocity ( $L A A-P A F V)$ was measured and recorded with TEE.

Results: Compared with baseline, the mean mitral E, septal E' and E'/A' ratio increased significantly after TAVI. In addition, the LAA-PAFV increased significantly within minutes of TAVI $(32.45 \pm 10.7 \mathrm{~cm} / \mathrm{s}$ vs. $47.6 \pm 12.6 \mathrm{~cm} / \mathrm{s}, p<0.001)$.

Conclusions: TAVI improves $L V$ diastolic function and LA performance immediately. (Cardiol J 2016; 23, 4: 449-455)

Key words: transcatheter aortic valve implantation, aortic valve stenosis, diastolic dysfunction, left atrial appendage function

Address for correspondence: Cenk Sarı, MD, Ankara Ataturk Education and Research Hospital, Department of Cardiology, Street: Bilkent, Postal code: 06800, Ankara, Turkey, tel: +90 3122912525, fax: +90 3122845652,

e-mail: cengaver61@yahoo.com

Received: 03.11.2015 Accepted: 16.05.2016 


\section{Introduction}

Aortic stenosis (AS) is the most commonly encountered valvular heart disease requiring surgery [1]. The raised pressure overload caused by AS, on left ventricle (LV) results in significant structural and functional changes, and affects the left atrium (LA) even before symptom development $[2,3]$. Transcatheter aortic valve implantation (TAVI) has emerged as a promising alternative to surgical aortic valve replacement for patients with severe AS and prohibitive or high surgical risk [4]. Several studies have already shown the immediate almost complete normalization in afterload and a significant decrease in pressure gradient, between the aorta and the LV, as well as clinical improvement at short-term follow-ups after TAVI $[5,6]$. However, so far, its acute impact on LA mechanics and LV diastolic function has not been demonstrated with the help of intra-procedural transesophageal echocardiography (TEE).

In this study, the authors' objective was to determine the acute effect of TAVI on LV diastolic function and LA performance, immediately after deployment of aortic bioprosthesis.

\section{Methods}

\section{Patients}

We prospectively enrolled 35 consecutive patients with severe symptomatic AS who underwent TAVI under TEE guidance in our clinic between July 2011 and March 2012. Operative risk of all patients was calculated based on Logistic European System for Cardiac Operative Risk Evaluation (EuroSCORE) [7] and the Society of Thoracic Surgeon (STS) score [8], and/or clinical judgement. Written informed consent of each patient was obtained and the Ethics Committee of our hospital approved the study. The decision to proceed with TAVI was made by a multidisciplinary heart team including invasive cardiologists, cardiovascular surgeons, radiologists, and anesthesiologists. Before the procedure, transthoracic echocardiography (TTE), TEE and multi-slice computed tomography (MSCT) of the subjects were performed to evaluate the convenience and technique of TAVI.

\section{Transthoracic echocardiography and Doppler measurements}

All patients underwent a comprehensive conventional TTE with 2-dimensional (2D), M-mode and Doppler assessment (IE33 echocardiography system, Philips Medical Systems, Eindhoven, the Neth- erlands) before the TAVI procedure. For all measurements, 3 beats in patients with sinus rhythm and 5 beats in patients with atrial fibrillation $(\mathrm{AF})$ were stored and off-line processed. All echocardiographic data were analyzed at the end of the data collection. Mean transvalvular gradient was calculated with Bernoulli formula and aortic valve area was measured by the continuity equation. Severe AS was defined as a mean transaortic pressure gradient $>40 \mathrm{~mm} \mathrm{Hg}$ or aortic valve area $<1 \mathrm{~cm}^{2}$ [9]. $\mathrm{LV}$ ejection fraction was calculated from apical views using the biplane Simpson's method. LV mass (LVM) was calculated by Devereux formula [10] and LVM index (LVMI) was calculated by dividing LVM by body surface area using Mosteller formula [11]. LV hypertrophy was defined as LVMI $>95 \mathrm{~g} / \mathrm{m}^{2}$ for women and LVMI $>115 \mathrm{~g} / \mathrm{m}^{2}$ for men. LA enlargement was determined by LA volume that was estimated by biplane $2 \mathrm{D}$ echocardiography using the modified Simpson's rule [12]. Normal LA volume indexed to body surface area was defined as $<34 \mathrm{~mL} / \mathrm{m}^{2}$ and LA enlargement was considered over this value.

To assess LV diastolic function from the beginning to the end of the procedure, modified American Society of Echocardiography recommendations with the use of the tissue Doppler parameters were used [13]. Pulsed-wave (PW) Doppler was performed in the apical 4-chamber view to obtain mitral inflow velocities to assess $\mathrm{LV}$ diastolic function. A 1-mm to 3-mm sample volume was placed between mitral leaflet tips during diastole, as recommended [13]. Measurements of mitral inflow included the peak of early filling wave (E), late diastolic filling wave (A) and the E/A ratio were obtained. $\mathrm{LV}$ diastolic function was also explored by pulsed tissue Doppler imaging (TDI), placing the sample volume at the level of mitral lateral and septal annulus from the apical 4-chamber view [14]. Early (E') and late (A') diastolic annular velocities were obtained. The E'/A' ratio was used as load-independent marker of LV diastolic relaxation [15]. The A and A' waves could not be obtained in patients with AF. Therefore $\mathrm{E} / \mathrm{A}, \mathrm{E}$ '/A', and septal A' were measured in 25 patients. The E/E' ratio was also obtained as a reliable index of LV filling pressures [16].

\section{Transcatheter aortic valve implantation}

Transcatheter aortic valve implantation was performed at the catheterization laboratory with fluoroscopy and TEE guidance and all under general anesthesia using conventional technique. Thirty-four (97\%) patients through femoral artery and 


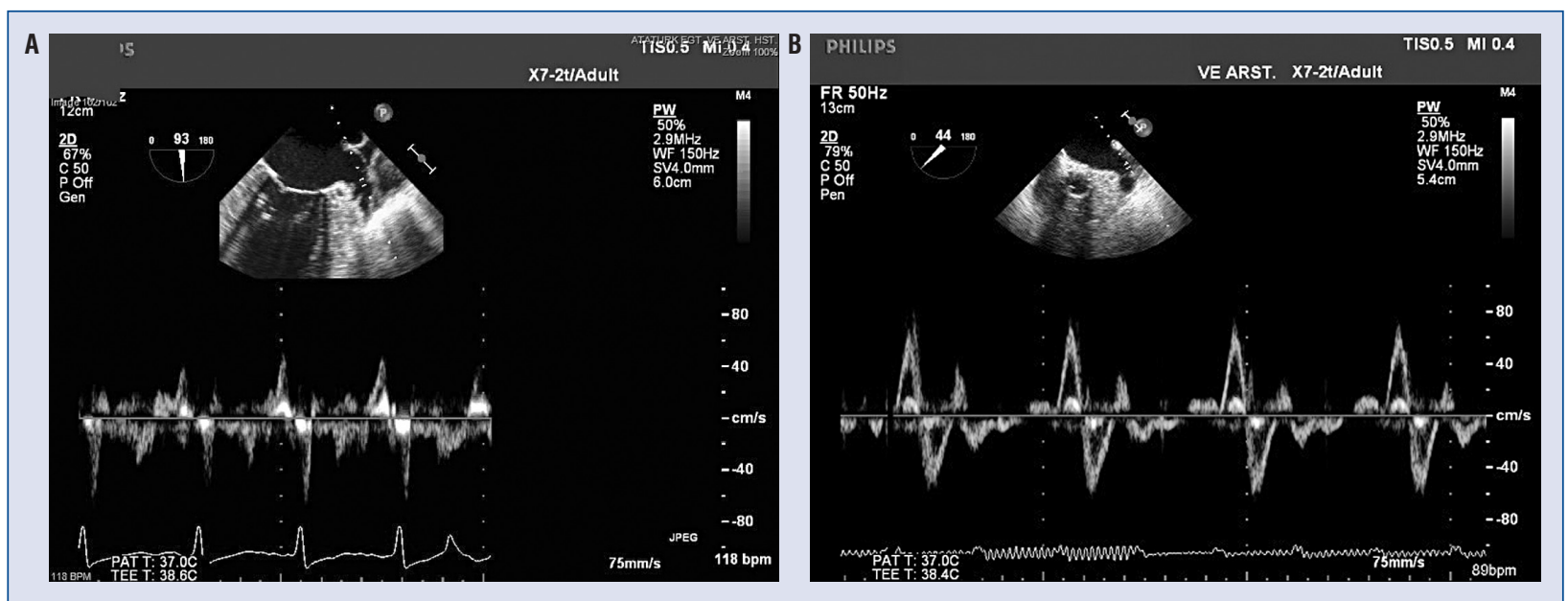

Figure 1. Left atrial appendage peak antegrade flow velocity measured during the procedure before (A) and minutes after (B) transcatheter aortic valve implantation with help of transesophageal echocardiography.

$1(3 \%)$ patient through subclavian artery received a balloon expandable Edwards SAPIEN (Edwards Lifesciences, Irvine, CA, USA) aortic valve. The sizes of the valves used were $23 \mathrm{~mm}$ and $26 \mathrm{~mm}$ and it was decided with combined multimodal imaging methods like TTE, TEE and MCST.

\section{Transesophageal echocardiography measurements}

During the procedure, left atrial appendagepeak antegrade flow velocity (LAA-PAFV) with TEE (IE33 echocardiography system, X7-2t probe, $7 \mathrm{MHz}$, Philips Medical Systems, Eindhoven, the Netherlands) was measured and recorded. TEE was performed just before the intervention and it was repeated few minutes after the valve deployment $(4.8 \pm 1.7 \mathrm{~min})$ to compare between TEE results before and after TAVI. PW spectral Doppler was recorded in mid-esophageal basal short-axis view at aortic valve level $\left(30-60^{\circ}\right)$ to assess the LAA-PAFV which was calculated by placing PW Doppler cursor in the LAA $2 \mathrm{~mm}$ from its orifice (Fig. 1). In sinus rhythm, LAA-PAFV was calculated as the average of the 3 highest velocities, whereas in patients with AF, the average of 5 cardiac cycles was used.

After the valve implantation, the presence of complications like cardiac effusion or tamponade, aortic regurgitation, valve displacement, LV wall motion abnormalities, and mitral valve injury were described [17]. Data expected to interfere with diastolic function parameters like systolic or diastolic blood pressure, heart rate, and hemoglobin level were assessed before the procedure and few minutes after the valve deployment.

\section{Statistical analysis}

Statistics were calculated using SPSS version 19.0 (IBM Corporation, Armonk, NY). Categorical variables were expressed as percentages and continuous variables as mean (standard deviation). A multivariate covariance analysis was also performed to evaluate the influence of potential confounders on main outcome variables. A probability value of $\mathrm{p}<0.05$ was considered statistically significant.

\section{Results}

\section{Baseline characteristics}

The baseline characteristic features of the patients were presented in Table 1 . Of the consecutive 35 study patients, $25(71.4 \%)$ were female. The mean age was $77.7 \pm 5.0$ years. The mean body mass index was $30.0 \pm 10.6 \mathrm{~kg} / \mathrm{m}^{2}$. Twenty-eight point six percent of patients had $\mathrm{AF}$ and $42 \%$ of patients had chronic renal failure (defined as glomerular filtration rate $<60 \mathrm{~mL} / \mathrm{min} / 1.73 \mathrm{~m}^{2}$ ) before the procedure. Also, pulmonary hypertension (defined as systolic pulmonary artery pressure $>40 \mathrm{~mm} \mathrm{Hg}$ ) was present in $48 \%$ of patients. According to the New York Heart Association classification, $74.3 \%$ of patients were in functional class III. The valve was successfully deployed in all patients.

\section{Conventional transthoracic and transesophageal echocardiography \\ All standard echocardiographic findings were shown in Table 2. After the valve deployment, the peak and mean transaortic systolic gradients, effec- tive orifice area, and LV ejection fraction improved}


Table 1. Baseline characteristic features of the patients.

\begin{tabular}{lc}
\hline Variables & $\mathrm{N}=35$ \\
\hline Age [years] & $77.7 \pm 5.0$ \\
Female & $71.4 \%$ \\
Body mass index $\left[\mathrm{kg} / \mathrm{m}^{2}\right]$ & $30.0 \pm 10.6$ \\
Logistic EuroSCORE $[\%]$ & $18.1 \pm 13.7$ \\
STS score [\%] & $7.0 \pm 3.7$ \\
Hypertension & $80 \%$ \\
Diabetes mellitus & $25.7 \%$ \\
Dyslipidemia & $45.7 \%$ \\
Coronary artery disease & $68.6 \%$ \\
Previous PCl & $20 \%$ \\
Prior CABG & $28.6 \%$ \\
Atrial fibrillation & $28.6 \%$ \\
NYHA functional class: & \\
I & $\mathrm{None}$ \\
II & $14.3 \%$ \\
III & $74.3 \%$ \\
IV & $11.4 \%$ \\
COPD & $55.9 \%$ \\
Renal impairment & $42 \%$ \\
Creatinine [mg/dL] & $1.0 \pm 0.41$ \\
Pulmonary hypertension & $48 \%$ \\
LA enlargement & $82.8 \%$ \\
Diastolic dysfunction* & $71.4 \%$ \\
Grade I & \\
Grade II & $34.3 \%$ \\
\hline Grade III & $20.0 \%$ \\
\hline Ting conpertrophy & $45.7 \%$ \\
\hline
\end{tabular}

*Grading concerns only the patients with sinus rhythm; CABG coronary artery bypass grafting; COPD - chronic obstructive pulmonary disease; EuroSCORE - European System for Cardiac Operative Risk Evaluation; LA - left atrial; LV - left ventricular; NYHA - New York Heart Association; PCI — percutaneous coronary intervention; STS - Society of Thoracic Surgeons

significantly. There were no statistically significant differences between the baseline examination and the examination after TAVI regarding LVMI, A wave, E/A ratio, septal A', and E/E' ratio. However, the parameters indicating early diastolic LV filling improved significantly: mean E $(1.0 \pm 0.38 \mathrm{~m} / \mathrm{s}$ vs. $1.2 \pm 0.34 \mathrm{~m} / \mathrm{s}$, respectively, $\mathrm{p}=0.003)$ and septal E' $(5.4 \pm 1.7 \mathrm{~cm} / \mathrm{s}$ vs. $6.9 \pm 2.4 \mathrm{~cm} / \mathrm{s}$, respectively, $\mathrm{p}=0.001$ ) increased. Furthermore, the load-independent marker of ventricular diastolic relaxation, E'/A' ratio increased significantly as well $(0.76 \pm$ \pm 0.21 vs. $1.02 \pm 0.42$, respectively, $\mathrm{p}<0.001)$.

When we analyzed the LAA-PAFV measured during the procedure with the help of TEE before and minutes after TAVI, we realized that it has been significantly improved after valve deployment $(32.45 \pm 10.7 \mathrm{~cm} / \mathrm{s}$ vs. $47.6 \pm 12.61 \mathrm{~cm} / \mathrm{s}$, respectively, $\mathrm{p}<0.001)$. In order to assess the influence of potential confounders on main outcome variables, a multivariate covariance analysis was performed. We found a significant increase in the $\mathrm{E}$ wave and LAA-PAFV in the study population (Table 3).

\section{Discussion}

In long-standing severe AS, a time-related structural and histologic remodeling occurs both in the LV, LA, and thereafter in the LAA. LV diastolic dysfunction caused by AS is well established $[18,19]$ and is related with LA dilatation which is assumed an indicator of severity and chronicity of diastolic dysfunction, as well as the magnitude of LA pressure rise [20-22]. During the diastolic period of left heart, the LA is directly exposed to LV pressure through the open mitral valve, thus it is affected by the chronic pressure rise resulting in increased LV filling pressures [23]. Therefore, many studies to date have come to the conclusion that the state of LA reflects the diastolic function of the LV [24, 25]. LA dysfunction in AS has been shown to predict mortality, indirectly enforcing the significance of raised LA pressure on the clinical outcome of patients with AS [26].

Decreased velocities of LAA in patients with AS can be observed in the presence of elevated LA pressure [27]. In addition, conditions that increase the LV filling pressure like AS impairs LAA flow [28]. Likewise, in patients with disturbed diastolic function, increasing $\mathrm{LV}$ end-diastolic pressure and LA pressure give rise to decrease in antegrade flow velocities of LAA [29]. Due to this fact, the loss of contractile elements of LAA may result in its reduced mechanical function which could be expressed in low LAA flow velocity profile. Various studies have proven LAA-PAFV to be a valuable means by which the structure and function of the LA may be predicted. More specifically, the studies have claimed that larger LA and LAA sizes are associated with lower LAA flow velocity [30]. TEE is the most widely used method for the evaluation of the LAA morphology and flow patterns within it. Therefore, in this study, we have used intra-procedural TEE to assess LAA-PAFV and we observed a significant increase in LAA-PAFV minutes after TAVI. Thus, we concluded that TAVI improves LA performance and this in turn improves LV diastolic function immediately. 
Table 2. Conventional echocardiographic results before and after the procedure $(n=35)$.

\begin{tabular}{|c|c|c|c|}
\hline Variables & Baseline & After TAVI & $\mathbf{P}$ \\
\hline \multicolumn{4}{|l|}{ Transthoracic echocardiographic variables } \\
\hline \multicolumn{4}{|l|}{ Aortic valve } \\
\hline Maximum systolic gradient [mm Hg] & $87.2 \pm 20.3$ & $18.2 \pm 4.3$ & $<0.001$ \\
\hline Mean systolic gradient [mm Hg] & $54.1 \pm 15.1$ & $9.1 \pm 2.4$ & $<0.001$ \\
\hline Effective orifice area $\left[\mathrm{cm}^{2}\right]$ & $0.62 \pm 0.16$ & $2.0 \pm 0.28$ & $<0.001$ \\
\hline Left ventricular ejection fraction [\%] & $54.4 \pm 12.7$ & $57.5 \pm 9.6$ & 0.039 \\
\hline Left ventricular mass index $\left[\mathrm{g} / \mathrm{m}^{2}\right]$ & $137.9 \pm 34.5$ & $138.4 \pm 28.5$ & 0.881 \\
\hline Degree of mitral regurgitation [\%]: & & & NS \\
\hline Mild & 68.6 & 84.4 & \\
\hline Moderate & 28.6 & 15.6 & \\
\hline Severe & 2.9 & None & \\
\hline $\mathrm{E}[\mathrm{m} / \mathrm{s}]$ & $1.0 \pm 0.38$ & $1.2 \pm 0.34$ & 0.003 \\
\hline At $[\mathrm{m} / \mathrm{s}]$ & $0.91 \pm 0.36$ & $0.92 \pm 0.4$ & 0.491 \\
\hline E/At ratio & $1.19 \pm 0.6$ & $1.2 \pm 0.6$ & 0.815 \\
\hline Septal E' $[\mathrm{cm} / \mathrm{s}]$ & $5.4 \pm 1.7$ & $6.9 \pm 2.4$ & 0.001 \\
\hline Septal $A^{\prime} \dagger[\mathrm{cm} / \mathrm{s}]$ & $7.1 \pm 3.8$ & $6.7 \pm 3.2$ & 0.641 \\
\hline$E^{\prime} / A^{\prime} \dagger$ ratio & $0.76 \pm 0.21$ & $1.02 \pm 0.42$ & $<0.001$ \\
\hline $\mathrm{E} / \mathrm{E}^{\prime}$ ratio & $20.1 \pm 8.1$ & $20.9 \pm 7.5$ & 0.718 \\
\hline \multicolumn{4}{|c|}{ Transesophageal echocardiographic variables } \\
\hline Degree of aortic regurgitation [\%]: & & & NS \\
\hline Absent or mild & 77.2 & 94.3 & \\
\hline Medium & 20 & 5.7 & \\
\hline Severe & 2.8 & None & \\
\hline Mean LAA-PAF [cm/s] & $32.45 \pm 10.7$ & $47.6 \pm 12.6$ & $<0.001$ \\
\hline
\end{tabular}

†Evaluated only in patients with sinus rhythm; A - atrial flow velocity; $\mathrm{A}^{\prime}$ - peak late diastolic velocity; E - mitral peak early diastolic velocity; $E^{\prime}$ - peak early diastolic velocity; LAA-PAF — left atrial appendage peak antegrade flow

Table 3. Covariance multivariate analysis of clinical parameters during the procedure $(n=35)$.

\begin{tabular}{|c|c|c|c|c|}
\hline Variables & Baseline $^{a}$ & Minutes after TAVI ${ }^{\mathrm{a}}$ & Difference of means (95\% Cl) & $\mathbf{P}$ \\
\hline E wave $[\mathrm{m} / \mathrm{s}]$ & $1.00(0.88 ; 1.12)$ & $1.09(0.9 ; 1.2)$ & $0.09(-0.17 ; 0.03)$ & $<0.001^{\mathrm{b}}$ \\
\hline LAA-PAF $[\mathrm{cm} / \mathrm{s}]$ & $32.4(28.7 ; 36.1)$ & $47.6(43.2 ; 51.9)$ & $15.2(-18.4 ;-11.7)$ & $<0.001^{\mathrm{b}}$ \\
\hline E/A ratio & $1.19(0.91 ; 1.47)$ & $1.24(0.9 ; 1.5)$ & $0.05(-0.38 ; 0.30)$ & 0.143 \\
\hline Septal E' $[\mathrm{cm} / \mathrm{s}]$ & $0.052(0.048 ; 0.057)$ & $0.053(0.050 ; 0.057)$ & $0.001(-0.006 ; 0.003)$ & 0.052 \\
\hline $\mathrm{E} / \mathrm{E}^{\prime}$ ratio & $20.1(17.3 ; 22.9)$ & $20.9(18.3 ; 23.6)$ & $0.08(-3.35 ; 2.33)$ & 0.718 \\
\hline$E^{\prime} / A^{\prime}$ ratio & $0.76(0.68 ; 0.84)$ & $1.02(0.93 ; 1.12)$ & $0.26(-0.32 ;-0.14)$ & $<0.001$ \\
\hline
\end{tabular}

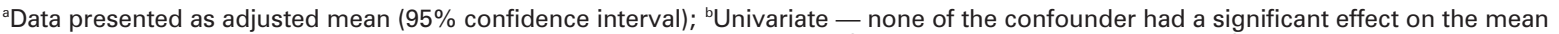
E wave and mean LAA-PAF variation during the procedure; LAA-PAF — left atrial appendix-peak antegrade flow

After TAVI, almost complete normalization of LV afterload occurs without significant variability among patients [5, 6]. Normalization of LV diastolic function [31] and benefit in ejection fraction, volumes, and hypertrophy are observed at long-term follow-up [32]. Gotzmann et al. [33] have determined improvement in diastolic function at 6-month follow-up in patients undergoing TAVI with CoreValve prosthesis.

The significant increase in LV E, E', and E'/A' ratio observed $24 \mathrm{~h}$ after TAVI and also significant increase in LAA-PAFV minutes after TAVI may 
imply an immediate improvement of LV diastolic function in our study. The increase in maximum $\mathrm{E}$ velocity may be explained by alterations in preload or in LV relaxation. The augmented LA-LV pressure during early diastole and the augmented $\mathrm{E}$ wave, are the consequence of an ameliorated LV relaxation, in response to immediate, complete and sustained afterload release.

Bauer et al. [34] demonstrated an increase in $\mathrm{E}^{\prime}$ and A' measured by pulsed TDI in LV septal wall but no difference in LV diastolic function measured by blood flow Doppler echocardiography when they examined the patients $24 \mathrm{~h}$ post-procedurally. After TAVI, our patients showed significant increases in effective orifice area and marked decreases in transvalvular pressure gradients, equivalent to a reduction in LV afterload [35]. In addition, E and E' increased significantly after TAVI. These results are consistent with previously published data from Gonçalves et al. [35] and Guarracino et al. [36], who measured LV relaxation parameters within minutes of TAVI.

Our main findings were those of immediate improvement of LV diastolic function and increase in LA hemodynamics in the minutes of valve deployment. TAVI gives rise to a unique opportunity regarding the assessment of $\mathrm{LV}$ diastolic function immediately after afterload release and the observed improvement is in concert with the expectations from the Frank-Starling law.

This study is, to the best of our knowledge, the first to examine the early recovery of LV diastolic function and LA performance after TAVI through comprehensive interpretation of echocardiographic findings including LAA-PAFV with TEE. We observed that after the release of chronic pressure overload with TAVI, an immediate improvement has occurred in diastolic function of the left heart. Our results shed light on the direct relationship between the mechanical function of LAA, LA and $\mathrm{LV}$ diastolic function in patients with severe AS.

\section{Limitations of the study}

The main limitation of our study is the limited number of patients. The reason for relatively small number of the patients conducted into our study is that we performed TAVI only to the first 35 patients with TEE. After that, due to no need for TEE guidance, other patients underwent TAVI without it.

\section{Conclusions}

From these results we concluded that LA and LAA functions decrease in patients with severe AS and LV diastolic dysfunction. TAVI results in early recovery of LA performance and LV diastolic function immediately after relieving $\mathrm{LV}$ afterload. An acute rise in LAA peak antegrade flow velocity is associated with improvement of LA mechanics and LV diastolic function. However, whether our results are persistent or transient is unknown and they should be evaluated with large scale studies.

\section{Conflict of interest: None declared}

\section{References}

1. Iung B, Baron G, Butchart EG et al. A prospective survey of patients with valvular heart disease in Europe: The Euro Heart Survey on Valvular Heart Disease. Eur Heart J, 2003; 24: 1231-1243 .

2. Lancellotti P, Moonen M, Magne J et al. Prognostic effect of longaxis left ventricular dysfunction and B-type natriuretic peptide levels in asymptomatic aortic stenosis. Am J Cardiol, 2010; 106: 1157-1162.

3. O’Connor K, Magne J, Rosca M, Pierard LA, Lancelotti P. Left atrial function and remodeling in aortic stenosis. Eur J Echocardiogr, 2011; 12: 299-305.

4. Zajarias A, Cribier AG. Outcomes and safety of percutaneous aortic valve replacement. J Am Coll Cardiol, 2009; 53: 1829-1836.

5. Grube E, Buellesfeld L, Mueller R et al. Progress and current status of percutaneous aortic valve replacement: Results of three device generations of the CoreValve Revalving system. Circ Cardiovasc Interv, 2008; 1: 167-175.

6. Clavel MA, Webb JG, Pibarot P et al. Comparison of the hemodynamic performance of percutaneous and surgical bioprosthesis fort he treatment of severe aortic stenosis. J Am Coll Cardiol, 2009; 53: 1883-1891.

7. Leontyev S, Walther T, Borger MA et al. Aortic valve replacement in octogenerians: Utility of risk stratification with EuroSCORE. Ann Thorac Surg, 2009; 87: 1440-1445.

8. Bagnall NM, Faiz O,Darzi A, Athanasiou T. What is the utility of preoperative frailty assessment for risk stratification in cardiac surgery? Intearct Cardiovasc Thorac Surg, 2013; 17: 398-402.

9. Vahanian A, Baumgartner H, Bax J et al.Task Force on the Management of Valvular Heart Disease of the European Society of Cardiology, ESC Committee for Practice Guidelines (2007). Guidelines on the management of valvular heart disease:the Task force on the Management of Valvular Heart Disease of the European Society of Cardiology. Eur Heart J, 2017; 28: 230-268.

10. Devereux RB, Reichek N. Echocardiographic determination of left ventricular mass in man. Anatomic validation of the method. Circulation, 1977; 55: 613-618.

11. Mosteller RD. Simplified calculation of body surface area. N Eng J Med, 1987; 317: 1098.

12. Lang RM, Badano LP, Mor-Avi V et al. Recommendations for chamber quantification by echocardiography in adults: An update from the American Society of Echocardiography and the European Association of Cardiovascular Imaging Eur Heart J Cardiovasc Imaging, 2015; 16: 233-271.

13. Nagueh SF, Appleton CP, Gillebert TC et al. Recommendations for the evaluation of left ventricular diastolic function by echocardiography. Eur J Echocardiogr, 2009; 10: 165-193.

14. Mor-Avi V, Lang RM, Badano LP et al. Current and evolving echocardiographic techniques fort he quantitative evaluation of cardiac mechanics: ASE/EAE consensus statement on methodology and indications endorsed by the Japanese Society of Echocardiography. Eur J Echocardiogr, 2011; 12: 167-205. 
15. Kasner M, Westermann D, Steendijk P et al. Utility of doppler echocardiography and tissue Doppler imaging in the estimation of diastolic function in heart failure with normal ejection fraction:a comparative Doppler-conductance catheterization study. Circulation, 2007; 116: 637-647.

16. Ommen SR, Nishimura RA, Appleton CP et al. Clinical utility of Doppler echocardiography and tissue Doppler imaging in the estimation of left ventricular filling pressures: A comparative simultaneous Doppler-catheterization study. Circulation, 2000; 102: 1788-1794.

17. Zoghbi WA, Enriquez-Sarano M, Foster E et al. Recommendations for evaluation of the severity of native valvular regurgitation with two-dimensional and Doppler echocardiography. J Am Soc Echocardiogr, 2003; 16: 777-802.

18. Gjertsson P, Caidahl K, Farasati M, Oden A, Bech-Hanssen O. Preoperative moderate to severe diastolic dysfunction: A novel Doppler echocardiographic long-term prognostic factor in patients with severe aortic stenosis. J Thorac Cardiovasc Surg, 2005; 129: 890-896.

19. Lund O, Flo C, Jensen FT et al. Left ventricular systolic and diastolic function in aortic stenosis: Prognostic value after valve replacement and underlying mechanisms. Eur Heart J, 1997; 18: 1977-1987.

20. Tsang T, Barnes ME, Gersh BJ, Bailey KR, Seward JB. Left atrial volume as a morphophysiologic expression of left ventricular diastolic dysfunction and relation to cardiovascular risk burden. Am J Cardiol, 2002; 90: 1284-1289.

21. Abhayaratna W, Seward JB, Appleton CP et al. Left atrial size: physiologic determinants and clinical applications. J Am Coll Cardiol, 2006; 47: 2357-2363.

22. Pritchett A, Mahoney DW, Jacobsen SJ, Rodeheffer RJ, Karon BL, Redfield MM. Diastolic dysfunction and left atrial volume: A population-based study. J Am Coll Cardiol, 2005; 45: 87-92.

23. Casaclang-Verzosa G, Malouf JF, Scott CG, Juracan EM, Nishimura RA, Pellikka PA. Does left atrial size predict mortality in asymptomatic patients with severe aortic stenosis? Echocardiography, 2010; 27: 105-109.

24. Kurt M, Wang J, Torre-Amione G, Nagueh SF. Left atrial function in diastolic heart failure. Circ Cardiovasc Imaging, 2009; 2: 10-15.

25. Pritchett AM, Mahoney DW, Jacobsen SJ, Rodeheffer RJ, Karon BL, Redfield MM. Diastolic dysfunction and left atrial volume: A population-based study. J Am Coll Cardiol, 2005; 45: 87-92.
26. Dahl JS, Videbæk L, Poulsen MK et al. Noninvasive assessment of filling pressure and left atrial pressure overload in severe aortic valve stenosis: Relation to ventricular remodeling and clinical outcome after aortic valve replacement. J Thorac Cardiovasc Surg 2011;142: e77-e83.

27. Tabata T, Oki T, Fukuda $\mathrm{N}$ et al. Influence of aging on left atrial appendage flow velocity patterns in normal subjects. J Am Soc Echocardiogr, 1996; 9: 274-280.

28. Ito T, Suwa M, Kobashi A, Yagi H, Hirota Y, Kawamura K. Influence of altered loading conditions on left atrial appendage function in vivo. Am J Cardiol, 1998; 81: 1056-1059.

29. Mullens W, Borowski AG, Curtin RJ, Thomas JD, Tang WH. Tissue Doppler imaging in the estimation of intracardiac filling pressure in decompansated patients with advanced systolic heart failure. Circulation, 2009; 119: 62-70.

30. Pollick C, Taylor D. Assessment of left atrial appendage function by transesophageal echocardiography: Implications fort he development of thrombus. Circulation, 1991; 84: 223-231.

31. Villari B, Vassalli G, Monrad ES, Chiariello M, Turina M, Hess OM. Normalization of diastolic dysfunction in aortic stenosis late after valve replacement. Circulation, 1995; 91: 2353-2358 .

32. Lund O, Erlandsen M. Changes in left ventricular function and mass during serial investigations after valve replacement for aortic stenosis. J Heart Valve Dis, 2000; 9: 583-593.

33. Gotzmann M, Lindstaedt M, Bojara W, Mugge A, Germing A. Hemodynamic results and changes in miyocardial function after transcatheter aortic valve implantation. Am Heart J, 2010; 159: 926-932.

34. Bauer F, Benigno S, Lemercier M et al. Early improvement of left ventricular function after implantation of a transcutaneous aortic valve: a tissue Doppler ultrasound study. Arch Cardiovasc Dis, 2009; 102: 311-318.

35. Gonçalves A, Marcos-Alberca P, Almeria C et al. Acute left ventricle diastolic function improvement after transcatheter aortic valve implantation. Eur J Echocardiogr, 2011; 12: 790-797.

36. Guarracino F, Talini E, Landoni G, Petronio S, Giannini C, Di Bello V. Effect of aortic valve surgery on left ventricular diastole assessed by echocardiography and neuroendocrine response: Percutaneous versus surgical approach. J Cardiothorac Vasc Anesth, 2010; 24: 25-29. 